\title{
COST OPTIMIZATION OF A CANTILEVER ROOF STRUCTURE FOR A SMALL FOOTBALL STADIUM IN LIMBUŠ, SLOVENIA
}

\author{
TOMAŽ ŽULA \& STOJAN KRAVANJA \\ Faculty of Civil Engineering, Transportation Engineering and Architecture, University of Maribor, Slovenia
}

\begin{abstract}
The paper deals with the cost optimization of a cantilever roof structure, designed for a small football stadium in Limbuš, located close to the city of Maribor, Slovenia. The structure was proposed to be designed from steel I sections for columns, beams and struts, and from reinforced concrete bases. The cost optimization of the structure was calculated by the Mixed-Integer Non-linear Programming (MINLP). The MINLP optimization model of the structure was developed. A cost objective function was applied and subjected to the design, resistance and deflection constraints. The dimensioning constraints were defined in accordance with Eurocodes 2 and 3. The Modified Outer-Approximation/ Equality-Relaxation (OA/ER) algorithm was used.

Keywords: cost optimization, roof structure, mixed-integer non-linear programming, MINLP.
\end{abstract}

\section{INTRODUCTION}

Many different methods for structural optimization have been developed and used in the near past. Kalanta et al. [1] have performed discrete optimization of steel bar structures by the branch and bound method. Cicconi et al. [2] have used virtual prototyping tools and the genetic algorithm to reduce the weight and cost of steel structures. Mela and Heinisuo [3] have introduced the particle swarm optimization method for the weight minimization and the minimum cost optimization of welded high strength steel beams. Recently, Hasançeb [4] has considered the sizing optimization of steel frames by the evolution strategy method.

This paper deals with the material, standard and rounded dimension optimization of a cantilever roof structure for the small football stadium in Limbuš, located five kilometers far from the city of Maribor, Slovenia. The optimization of the cantilever is calculated by the Mixed-Integer Non-linear Programming (MINLP). The MINLP performs the discrete standard dimension optimization of columns, beams and struts (standard sizes optimization) as well as the rounded dimension optimization of reinforced concrete bases (the bases' dimensions are rounded on whole 10 centimeters) simultaneously with the continuous optimization of the structure self-manufacturing costs, internal forces and deflections.

The task of the optimization is to minimize the self-manufacturing costs of the cantilever roof structure. A cost objective function is applied and subjected to the design, resistance and deflection constraints. The dimensioning constraints are defined in accordance with Eurocode 3 [5] for steel, Eurocode 2 [6] for concrete and Eurocode 7 [7] for geotechnics.

The Modified Outer-Approximation/Equality-Relaxation algorithm is applied, see Kravanja and Grossmann [8], and Kravanja et al. [9], [10]. A three-phase MINLP optimization is used. The calculation begins with the overall continuous optimization. The first result is used as the starting point for the further second step, where the discrete optimization of steel grades is executed (standard and rounded dimensions are still continuous). After the optimal steel grade is obtained, the overall discrete material, standard and rounded dimension optimization of steel sections and concrete base is calculated. 


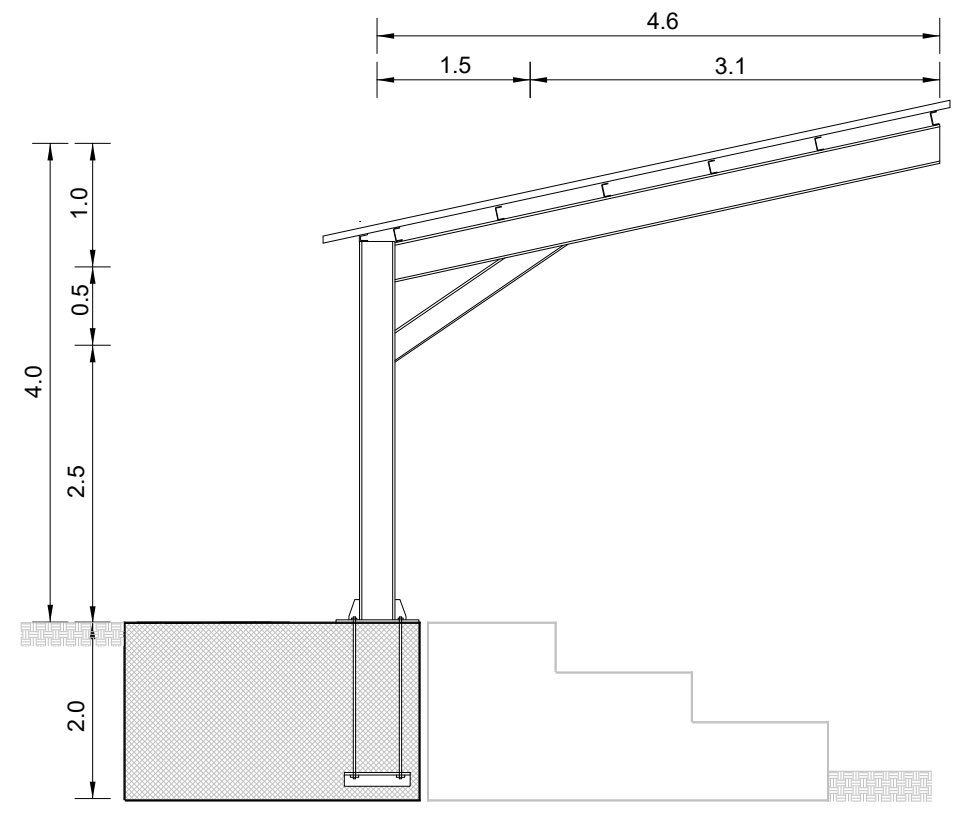

Figure 1: Cantilever roof structure.

\section{CANTILEVER ROOF STRUCTURE}

The paper presents the material, standard and rounded dimension optimization of a steel cantilever roof structure for the small football stadium in Limbuš. The treated steel structure is 16.0 metres long, 4.0 metres high and 4.6 metres wide. The structure is consisted from four equal cantilever frames with the intermediate distances of 5.33 meters, clamped onto four reinforced concrete bases (Fig. 1). The cantilever frame structure is proposed to be designed from standard hot rolled steel I sections for columns, beams and struts. Purlins which are mutually connected onto cantilever frames are not included in the optimization. Each reinforced concrete base is 2.0 meters high and 1.5 meters wide, placed behind the existed concrete seat structure. The in-plane length of the base $L$ is treated in the optimization as a variable.

The cantilever frame structure is subjected to self-weight $g$ and uniformly distributed variable load $q$ ( snow $s=1.5 \mathrm{kN} / \mathrm{m}^{2}$ and wind $w=0.14 \mathrm{kN} / \mathrm{m}^{2}$ ). The design loads are defined: $1.35 \cdot g+1.50 \cdot s+1.50 \cdot 0.6 \cdot w$ for ultimate limit states, and $1.00 \cdot g+1.00 \cdot s+1.00 \cdot 0.6 \cdot w$ for serviceability limit states. Constraints for internal forces and deflections are defined by the force method. Eurocodes 2, 3 and 7 are used. When the ultimate limit state of structural elements is considered, the steel members are checked for:

- Axial resistance, see eqn (1). $N_{E d}$ stands for the design axial force, $A$ represents the crosssection area of the member, $f_{y}$ is the yield strength of steel and $\gamma_{M 0}$ is the resistance partial safety factor

$$
N_{E d} \leq \frac{A \cdot f_{y}}{\gamma_{M 0}} .
$$

- Shear resistance, see eqn (2), where $V_{E d}$ denotes the design shear force and $A_{v}$ is the effective shear area of cross-sections 


$$
V_{E d} \leq A_{v} \cdot \frac{f_{y}}{\sqrt{3}} \cdot \frac{1}{\gamma_{M 0}} .
$$

- $\quad$ Bending moment resistance, see eqn (3). $M_{E d}$ represents the design bending moment and $W$ is the section modulus of the steel member

$$
M_{E d} \leq \frac{W \cdot f_{y}}{\gamma_{M 0}} .
$$

- Compression/buckling resistance, determined by eqn (4), $\chi$ is the reduction factor due to the flexural buckling and $\gamma_{M 1}$ is the resistance partial safety coefficient

$$
N_{E d} \leq \chi \frac{A \cdot f_{y}}{\gamma_{M 1}} .
$$

- The interaction between the compression and bending moment resistance, defined by eqn (5)

$$
\frac{N_{E d}}{A \cdot f_{y} / \gamma_{M 0}}+\frac{M_{E d}}{W \cdot f_{y} / \gamma_{M 0}} \leq 1.0
$$

- The interaction between the buckling and the lateral-torsional buckling resistance, checked by eqn (6), where $\chi_{L T}$ is the reduction factor due to the lateral-torsional buckling

$$
\frac{N_{E d}}{\chi \cdot A \cdot f_{y} / \gamma_{M 1}}+\frac{M_{E d}}{\chi_{L T} \cdot W \cdot f_{y} / \gamma_{M 1}} \leq 1.0 .
$$

- The bearing resistance failure in the ground under the reinforced concrete bases, checked by eqn (7). $N$ presents the vertical force and $M$ is the bending moment. $A_{b}, L$ and $b_{b}$ stand for the surface area, length and width of the base, respectively. While the surface area is calculated by eqn (8), the section modulus of the base $W_{b}$ is defined by eqn (9)

$$
\begin{gathered}
\sigma=\frac{N}{A_{b}}+\frac{M}{W_{b}} \leq 200 k P a, \\
A_{b}=L \cdot b_{b}, \\
W_{b}=\frac{b_{b} \cdot L^{2}}{6} .
\end{gathered}
$$

- Overturning resistance of the reinforced concrete base, see eqn (10), where $G$ is the selfweight of the reinforced concrete base, $F_{\text {tot }}$ is the total action force (the self-weight plus snow plus wind), $e$ is the distance between the total force and the point of overturning (the right bottom base edge) and $\gamma$ is the safety factor $(\gamma=2.5)$

$$
G \cdot \frac{L}{2} \geq F_{\text {tot }} \cdot e \cdot \gamma
$$


When the serviceability limit state is verified, the cantilever total vertical deflection $\delta$ is checked by eqn (11), where $L_{c r}$ is the span of cantilever roof structure

$$
\delta \leq \frac{2 \cdot L_{c r}}{200} .
$$

\section{MINLP MODEL FORMULATION}

Since the problem of the roof structure is the non-linear discrete-continuous optimization problem, the MINLP is applied for the solution. The general MINLP optimization problem can be formulated as follows:

$$
\begin{aligned}
& \min \quad z=\mathbf{c}^{\mathrm{T}} \mathbf{y}+\mathrm{f}(\mathbf{x}) \\
& \text { s.t. } \\
& \mathbf{g}(\mathbf{x}) \leq \mathbf{0} \\
& \mathbf{B y}+\mathbf{C x} \leq \mathbf{b} \\
& \mathbf{x} \in X=\left\{\mathbf{x} \in R^{n}: \mathbf{x L O} \leq \mathbf{x} \leq \mathbf{x U P}\right\} \\
& \mathbf{y} \in Y=\{0,1\}^{m} \text {, }
\end{aligned}
$$

where $\mathbf{x}$ is a vector of continuous variables and $\mathbf{y}$ is a vector of discrete/binary $0-1$ variables. While $f(\mathbf{x})$ presents a dimension dependent non-linear function in the objective, $\mathbf{c}^{\mathrm{T}} \mathbf{y}$ is linear fixed item, $\mathbf{g}(\mathbf{x})$ stand for non-linear functions in the (in)equality constraints. $\mathbf{B y}+\mathbf{C x} \leq \mathbf{b}$ are mixed linear (in)equality constraints.

The general MINLP-G model formulation is extended for the optimization of mechanical structures (MINLP-MS). The MINLP-MS model formulation is defined as follows:

$$
\begin{gathered}
\min z=\mathbf{c}^{\mathrm{T}} \mathbf{y}+\mathrm{f}(\mathbf{x}) \\
\text { s.t. } \\
\mathbf{g}(\mathbf{x}) \leq \mathbf{0} \\
\mathbf{A}(\mathbf{x}) \leq \mathbf{a} \\
\mathbf{E} \mathbf{y} \leq \mathbf{e} \\
\mathbf{P y}+\mathbf{M}\left(\mathbf{d}^{\mathrm{mat}}\right) \leq \mathbf{m} \\
\mathbf{P y}+\mathbf{S}\left(\mathbf{d}^{\mathrm{st}}\right) \leq \mathbf{s} \\
\mathbf{P y}+\mathbf{R}\left(\mathbf{d}^{\mathrm{rd}}\right) \leq \mathbf{r} \\
\mathrm{x} \in X=\left\{\mathbf{x} \in R^{n}: \mathrm{xLO} \leq \mathrm{x} \leq \mathrm{xUP}\right\} \\
\mathbf{y} \in Y=\{0,1\}^{m},
\end{gathered}
$$

where $\mathbf{g}(\mathbf{x}) \leq \mathbf{0}$ and $\mathbf{A}(\mathbf{x}) \leq \boldsymbol{a}$ stand for the design, resistance and deflection constraints, $\mathbf{E y} \leq$ e define the relations between binary variables, $\mathbf{P y}+\mathbf{M}\left(\mathbf{d}^{\text {mat }}\right) \leq \mathbf{m}$ determine discrete materials (grades), $\mathbf{P y}+\mathbf{S}\left(\mathbf{d}^{\text {st }}\right) \leq \mathbf{s}$ calculate standard dimensions and $\mathbf{P y}+\mathbf{R}\left(\mathbf{d}^{\text {rd }}\right) \leq \mathbf{r}$ define rounded dimensions (length of the concrete base is rounded up to whole 10 centimetres). See the formulation in detail in [11], [12].

\section{MINLP OPTIMIZATION}

The optimization of the structure is carried out by the MINLP computer program MIPSYN, the extension of PROSYN by Kravanja and Grossmann [8]. The optimization model is modelled according to the above model formulation with GAMS (General Algebraic 
Modelling System) by Brooke et al. [13]. The Modified OA/ER algorithm and the threephased optimization are applied. While GAMS/CONOPT2 (Generalized Reduced-Gradient method) by Drudd [14] is used to solve NLP problems, GAMS/CPLEX [15] (Branch and Bound) is used to solve MILP problems.

The cost objective function comprises the self-manufacturing costs of the material, anticorrosion resistant painting, panelling, connections and assembling, see eqn (12). Table 1 presents the defined material and labour prices/costs.

$$
\begin{aligned}
\min : \operatorname{COST}= & C_{\mathrm{c}} \cdot V_{\mathrm{c}}+C_{\mathrm{s}} \cdot \rho_{\mathrm{s}} \cdot V_{\mathrm{s}}+C_{\mathrm{r}} \cdot \rho_{\mathrm{s}} \cdot V_{\mathrm{r}}+C_{\mathrm{ac}} \cdot A_{\mathrm{ac}}+C_{\mathrm{sc}}+ \\
& 0.15 \cdot C_{\mathrm{s}} \cdot \rho_{\mathrm{s}} \cdot V_{\mathrm{s}}+C_{\mathrm{p}} \cdot V_{\mathrm{p}}+C_{\mathrm{pl}} \cdot A_{\mathrm{pl}},
\end{aligned}
$$

where COST denotes the self-manufacturing costs of the cantilever roof structure; $C_{\mathrm{c}}, C_{\mathrm{s}}, C_{\mathrm{r}}$, $C_{\mathrm{ac}}, C_{\mathrm{sc}}, C_{\mathrm{p}}$ and $C_{\mathrm{pl}}$ are the prices of the concrete and steel materials, of the anti-corrosion resistant painting, of the steel connections as well as the prices of the construction pit and of the panelling, see Table $1 . V_{\mathrm{c}}, V_{\mathrm{s}}, V_{\mathrm{r}}$ and $V_{\mathrm{p}}$ represent the volumes of concrete base, steel I sections, reinforcing steel and excavation, respectively; $\rho_{\mathrm{s}}$ is the unit mass of steel; $A_{\mathrm{ac}}$ is the exposed area of steel members; and $A_{\mathrm{pl}}$ is the area of panelling. Assembling costs of the steel structure is defined to be $15 \%$ of the calculated material costs.

The cantilever roof superstructure includes different material, standard dimension and rounded dimension discrete alternatives. The superstructure comprises 3 different structural steel grades (S235, S275, S355), 24 different standard hot rolled European wide flange sections (from HEA 100 to HEA 1000) for column, 18 different standard hot rolled European I sections (from IPE 80 to IPE 600) separately for beam and strut, and 61 various discrete alternatives for rounding up on whole 10 centimeters (from 100 to $700 \mathrm{~cm}$ ) for the length of the concrete base.

The optimal discrete design of the cantilever roof structure is gained in the 24th MINLP iteration. The minimal self-manufacturing costs of $4545 €$ per cantilever frame are obtained, and $18180 €$ for all four structures (the selling price is approx. 2.5 times higher than these production costs). The calculated optimal design comprises the structural steel grade S355, steel sections HEA 400 for columns, sections IPE 300 for beams and IPE 200 for struts. Calculated is also the concrete base length of $540 \mathrm{~cm}$. The calculated design is shown in Fig. 2. The results obtained with the optimization yielded $24 \%$ of savings in steel and $30 \%$ in concrete when compared to the classical structural analysis calculations.

\section{CONCLUSIONS}

The paper presents the cost optimization of a cantilever roof structure, designed for the small football stadium in Limbuš, located close to the city of Maribor, Slovenia. The MINLP optimization model of the roof structure and the cost objective function were developed. The optimization was performed by the MINLP computer program MIPSYN. The Modified $\mathrm{OA} / \mathrm{ER}$ algorithm and the three-phased optimization strategy were applied.

Table 1: Material and labour costs.

\begin{tabular}{|l|l|l|}
\hline$C_{\mathrm{c}}$ Material and erection costs for concrete C 25/30 & 125.0 & $\mathrm{EUR} / \mathrm{m}^{3}$ \\
\hline$C_{\mathrm{s}}$ Material costs for structural steel S 235 & 1.25 & $\mathrm{EUR} / \mathrm{kg}$ \\
\hline$C_{\mathrm{r}}$ Material costs for reinforcing steel B 500 & 1.30 & $\mathrm{EUR} / \mathrm{kg}$ \\
\hline$C_{\mathrm{ac}}$ Anti-corrosion resistant painting costs & 20.0 & $\mathrm{EUR} / \mathrm{m}^{2}$ \\
\hline$C_{\mathrm{sc}}$ Costs per steel connection & 300.0 & $\mathrm{EUR}$ \\
\hline$C_{p}$ Construction pit (incl. excavation, removal, planning) & 10.0 & $\mathrm{EUR} / \mathrm{m}^{3}$ \\
\hline$C_{\mathrm{pl}}$ Panelling costs (incl. material and erection) & 5.0 & $\mathrm{EUR} / \mathrm{m}^{2}$ \\
\hline
\end{tabular}




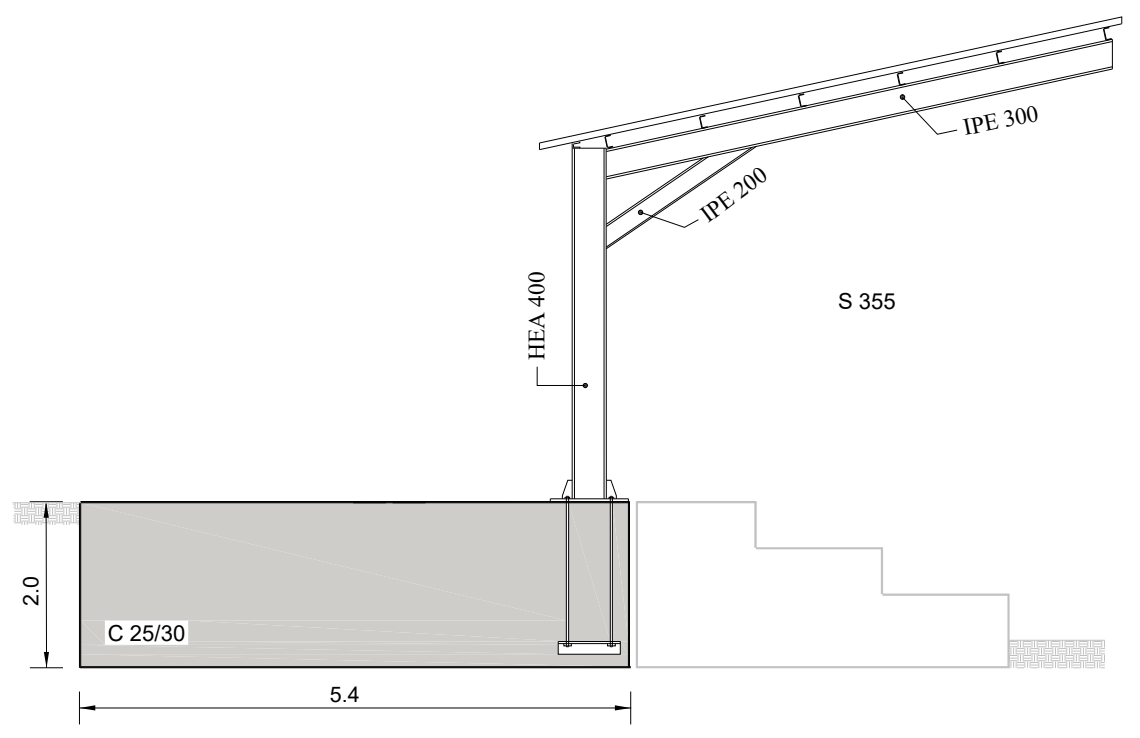

Figure 2: Optimum design of the cantilever roof structure.

The obtained optimal/minimal self-manufacturing costs of the structure represent $24 \%$ of savings in steel and $30 \%$ in concrete when compared to the classical structural analysis calculations. The example clearly shows the advantage of the proposed MINLP optimization approach.

\section{ACKNOWLEDGEMENT}

The authors are grateful for the support of funds from the Slovenian Research Agency (program P2-0129).

\section{REFERENCES}

[1] Kalanta, S., Atkočiunas, J. \& Venskus, A., Discrete optimization problems of the steel bar structures. Engineering Structures, 31, pp. 1298-1304, 2009.

[2] Cicconi, P., Germani, M., Bondi, S., Zuliani, A. \& Cagnacci, E., A design methodology to support the optimization of steel structures. Procedia CIRP, 50, pp. 58-64, 2016.

[3] Mela, K. \& Heinisuo, M., Weight and cost optimization of welded high strength steel beams. Engineering Structures, 79(3), pp. 54-64, 2014.

[4] Hasançeb, O., Cost efficiency analyses of steel frameworks for economical design of multi-storey buildings. JCSR, 128, pp. 380-396, 2017.

[5] Eurocode 3, Design of steel structures, European Committee for Standardization: Brussels, 2005.

[6] Eurocode 2, Design of concrete structures, European Committee for Standardization: Brussels, 2004.

[7] Eurocode 7, Geotechnical design, European Committee for Standardization: Brussels, 2004. 
[8] Kravanja, Z. \& Grossmann, I.E., New developments and capabilities in PROSYN: An automated topology and parameter process synthesizer. Computers Chem. Eng., 18, pp. 1097-1114, 1994.

[9] Kravanja, S., Kravanja, Z. \& Bedenik, B.S., The MINLP optimization approach to structural synthesis. Part I: A general view on simultaneous topology and parameter optimization. Int. J. Numer. Methods Eng., 43, pp. 263-292, 1998.

[10] Kravanja, S., Kravanja, Z. \& Bedenik, B.S., The MINLP optimization approach to structural synthesis. Part II: Simultaneous topology, parameter and standard dimension optimization by the use of the Linked two-phase MINLP strategy. Int. J. Numer. Methods Eng., 43, pp. 293-328, 1998.

[11] Kravanja, S., Silih, S. \& Kravanja, Z., The multilevel MINLP optimization approach to structural synthesis: the simultaneous topology, material, standard and rounded dimension optimization. Advances in Engineering Software, 36(9), pp. 568-583, 2005.

[12] Žula, T. \& Kravanja, S., The two-phase MINLP optimization of a single-storey industrial steel building. High Performance Structures and Materials IV, pp. 439-448, 2008.

[13] Brooke, A., Kendrick, D. \& Meeraus, A., GAMS: A User Guide, Scientific Press: Redwood City, CA, 1988.

[14] Drudd, A.S., CONOPT: A large-scale GRG Code. ORSA J. Comput. 6, pp. 207-216, 1994.

[15] CPLEX 11 User Manuals, ILOG Inc., 2007. 